Abbas Bahri

\title{
A remark on the bifurcation diagrams of superlinear elliptic equations
}

\section{Dedicated to Antonio Ambrosetti on his sixtieth birthday}

Received October 27, 2005

Abstract. We prove a formula relating the index of a solution and the rotation number of a certain complex vector along bifurcation diagrams.

We consider a deformation $\Omega_{t}$ of domains via uniform dilation. For the sake of simplicity, we will consider only the case of starshaped domains.

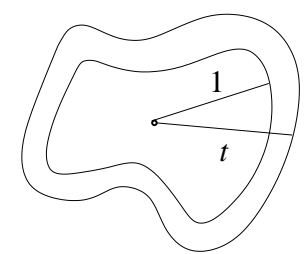

On $\Omega_{t}$, we consider the partial differential equation

$$
\left\{\begin{array}{l}
-\Delta u=g(u), \\
\left.u\right|_{\partial \Omega_{t}}=0 .
\end{array}\right.
$$

where $g(u)$ is "superlinear" and "subcritical", i.e., $g: \mathbb{R} \rightarrow \mathbb{R}$ and

$$
\lim _{|s| \rightarrow \infty} \frac{g(s)}{s}=+\infty, \quad|g(s)| \leq C\left(1+|s|^{q}\right) \quad \text { with } q<\frac{n+2}{n-2} \quad(n \geq 3) .
$$

We assume that $g$ is $C^{\infty}$ for the sake of simplicity.

For a generic shape of domains $\Omega_{1}$, we may assume that the solution set $\left(t, u_{t}\right), t \in$ $(0, \infty)$, is a one-dimensional manifold having possibly infinitely many connected components.

A natural question is: Does every connected component span over $t \in(0, \infty)$ ? Are there infinitely many components in the solution set spanning over $(0, \infty)$ ?

Both questions are reformulations of the following conjecture:

Conjecture. For any given $t_{0},(1)$ has infinitely many solutions.

A. Bahri: Department of Mathematics, Rutgers University, 110 Frelinghuysen Road, Piscataway, NJ 08854-8019, USA; e-mail: abahri@math.rutgers.edu 
A related problem is the following. Let $0<a<b$ be given. Are the connected components for $t \in[a, b]$ compact? i.e., assuming that we are considering a branch of solutions $u_{t}, t \in[a, b]$, of

$$
\left\{\begin{array}{l}
-\Delta u_{t}=g\left(u_{t}\right) \\
\left.u_{t}\right|_{\partial \Omega_{t}}=0
\end{array}\right.
$$

is the Morse index of $u_{t}$ bounded on a given connected component for $t \in[a, b]$ ?

Indeed, by the results of X. F. Yang [2] and Harrabi-Rebhi-Selmi [1], a bound on the Morse index of $u_{t}$ is equivalent to a bound on $\left\|u_{t}\right\|_{\infty}$ for $t \in[a, b]$ under the additional assumptions:

(i) $g(u) \underset{|u| \rightarrow \infty}{\sim} c_{+}\left(u^{+}\right)^{p_{+}}-c_{-}\left(u^{-}\right)^{p_{-}}, 1<p_{+}, p_{-}<(n+2) /(n-2)$,

(ii) $g^{\prime}(u) \underset{|u| \rightarrow \infty}{\sim} p_{+} c_{+}\left(u^{+}\right)^{p_{+}-1}-p_{-} c_{-}\left(u^{-}\right)^{p_{-}-1}$.

Let us consider such a connected component:

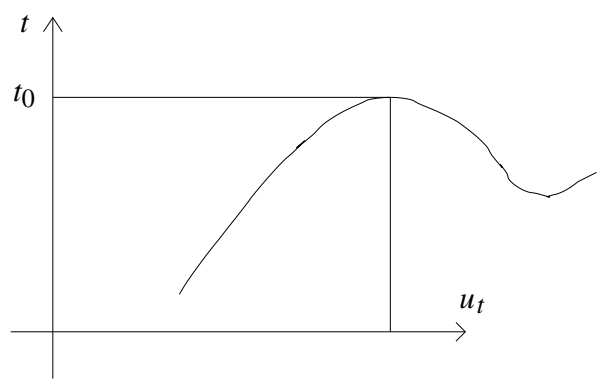

For values of $t$ such as $t=t_{0},(1)$ degenerates at $u_{t_{0}}$ and the Morse index of $u_{t}$ changes.

Picking up two points $\left(t_{1}, u_{t_{1}}\right)$ and $\left(t_{2}, u_{t_{2}}\right)$ on $\mathcal{C}$, we would like to relate the Morse index of $u_{t_{2}}$ to the Morse index of $u_{t_{1}}$.

We introduce the vector $(\mathcal{C}$ is parametrized by $s)$ :

$$
V(s)=\int_{\Omega_{t(s)}}\left|\nabla u_{t(s)}^{s}\right|^{2}+i \int_{\Omega_{t(s)}} G\left(u_{t(s)}^{s}\right) \quad \text { with } \quad G(u)=\int_{0}^{u} g(x) d x .
$$

We claim that:

Theorem 1. $\dot{V}(s)$ is never zero on $\mathcal{C}$ generically on $\Omega_{1}$ and

Morse index $\left(u_{t_{2}}\right)-$ Morse index $\left(u_{t_{1}}\right)$

$$
=\text { algebraic number of times } \dot{V}(s) \text { crosses the } y \text {-axis. }
$$

Proof. Let us differentiate (1) with respect to $s$. We derive

$$
\left\{\begin{array}{l}
-\Delta h=g^{\prime}(u) h, \\
h+\left.\operatorname{tr}(\sigma) \frac{\partial u}{\partial r}(\sigma, \operatorname{tr}(\sigma))\right|_{\partial \Omega_{t}}=0 .
\end{array}\right.
$$

with $\partial \Omega_{1}$ parametrized by $(\sigma, r(\sigma)), \sigma \in S^{n-1}$. 
Indeed, the Dirichlet boundary condition reads $u_{t}(\sigma, \operatorname{tr}(\sigma))=0$ and we derive our boundary condition after differentiation.

The Morse index changes only when $\dot{t}$ vanishes, so that we have

$$
\left\{\begin{array}{l}
-\Delta h=g^{\prime}(u) h, \\
\left.h\right|_{\partial \Omega}=0 .
\end{array}\right.
$$

Observe that, with $I_{t}(u)=\frac{1}{2} \int_{\Omega_{t}}|\nabla u|^{2}-\int_{\Omega_{t}} G(u)$, we find

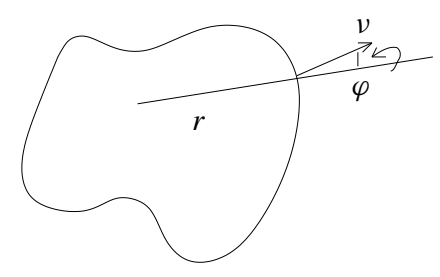

$$
\begin{aligned}
& \frac{d}{d s} I_{t(s)}\left(u^{s}\right) \\
& \quad=\int_{\Omega_{t}} \nabla u^{s} \nabla h-\int_{\Omega_{t}} g\left(u^{s}\right) h+\frac{d}{d s}\left(\int_{0}^{t}\left(\int_{\partial \Omega_{x}} \frac{\left|\nabla u^{x}\right|^{2}}{2} d \sigma_{x}\right) \cos \varphi(\sigma) r(\sigma) d y\right) \\
& =\int_{\partial \Omega_{t}} \frac{\partial u^{s}}{\partial v} h+\frac{\dot{t}}{2} \int_{\partial \Omega_{t}}\left|\nabla u^{s}\right|^{2} r(\sigma) \cos \varphi(\sigma) d \sigma_{t} \\
& =-\dot{t} \int_{\partial \Omega_{t}} \frac{\partial u^{s}}{\partial v} \frac{\partial u^{s}}{\partial r} r d \sigma_{t}+\frac{\dot{t}}{2} \int_{\partial \Omega_{t(s)}}\left|\frac{\partial u}{\partial v}\right|^{2} \cos \varphi(\sigma) r(\sigma) d \sigma_{t} \\
& =-\frac{i}{2} \int_{\partial \Omega_{t}}\left|\nabla u^{s}\right|^{2} r(\sigma) r(\sigma) \cos \varphi(\sigma) d \sigma_{t} .
\end{aligned}
$$

On the other hand, if $\dot{t}\left(s_{0}\right)=0$, we compare $I_{t}\left(u_{+}\right)$and $I_{t}\left(u_{-}\right)$, where $u_{+}$and $u_{-}$are solutions for $s_{0}+k, k>0$ small, and $s_{0}-k_{1}, k_{1}>0$ small, with

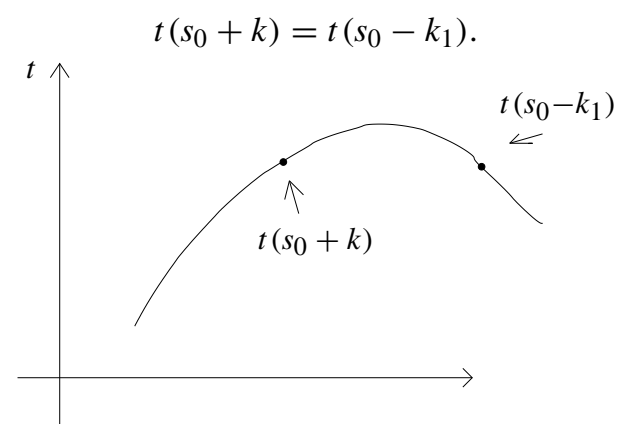

This will tell us how the Morse index changes as $s$ increases because whichever of $I_{t\left(s_{0}+k\right)}\left(u\left(s_{0}+k\right)\right)$ or $I_{t\left(s_{0}-k_{1}\right)}\left(u\left(s_{0}-k_{1}\right)\right)$ is larger will correspond to the larger index: 
when an elimination of a pair of critical points occurs in a variational problem, the highest index critical point is above the lowest one.

We renormalize $\Omega_{t(s)}$ near $s=s_{0}$ so that we will be considering only one $\Omega_{t\left(s_{0}\right)}=\Omega_{0}$ with a functional

$$
\widetilde{I}_{t(s)}=t(s)^{n-2} \bar{I}_{t(s)}\left(u\left(\frac{x}{t(s)}\right)\right) \quad\left(t\left(s_{0}\right)=1 \text { for example }\right) .
$$

Our critical points $u\left(s_{0}+k\right)$ and $u\left(s_{0}-k_{1}\right)$ change into $\tilde{u}\left(s_{0}+k\right)$ and $\tilde{u}\left(s_{0}-k_{1}\right)$. We know that $\dot{t}\left(s_{0}\right)=0$.

The branch $(t(s), \tilde{u}(s))$ is differentiable. With $\dot{\tilde{u}}\left(s_{0}\right)=h$, the direction of degeneracy, we have

$$
\left\{\begin{array}{l}
\tilde{u}\left(s_{0}+k\right)=u\left(s_{0}\right)+k h+O\left(k^{2}\right), \\
\tilde{u}\left(s_{0}-k_{1}\right)=u\left(s_{0}\right)-k_{1} h+O\left(k_{1}^{2}\right), \\
t\left(s_{0}+k\right)=t\left(s_{0}-k_{1}\right) .
\end{array}\right.
$$

Let $w=\tilde{u}\left(s_{0}+k\right)-\tilde{u}\left(s_{0}-k_{1}\right)$. We expand

$$
\begin{aligned}
\Delta= & \widetilde{I}_{t\left(s_{0}+k\right)}\left(\tilde{u}\left(s_{0}+k\right)\right)-\widetilde{I}_{t\left(s_{0}-k_{1}\right)}\left(\tilde{u}\left(s_{0}-k_{1}\right)\right) \\
= & t\left(s_{0}-k_{1}\right)^{n-2}\left(\bar{I}_{t\left(s_{0}+k\right)}\left(\tilde{u}\left(s_{0}+k\right)\right)-\bar{I}_{t\left(s_{0}-k_{1}\right)}\left(\tilde{u}\left(s_{0}-k_{1}\right)\right)\right)=t\left(s_{0}-k_{1}\right)^{n-2} \bar{\Delta}, \\
\bar{\Delta}= & \bar{I}_{t\left(s_{0}+k\right)}\left(\tilde{u}\left(s_{0}+k\right)\right)-\bar{I}_{t\left(s_{0}-k_{1}\right)}\left(\tilde{u}\left(s_{0}-k_{1}\right)\right)=\frac{1}{2} \bar{I}_{t\left(s_{0}+k\right)}^{\prime \prime}\left(u\left(s_{0}-k_{1}\right)\right) \cdot w \cdot w \\
& +\frac{1}{6} \bar{I}_{t\left(s_{0}+k\right)}^{(3)}\left(u\left(s_{0}-k_{1}\right)\right) \cdot w \cdot w \cdot w+\frac{1}{4} \bar{I}^{(4)}\left(u\left(s_{0}-k_{1}\right)\right) w \cdot w \cdot w \cdot w+O\left(|w|_{H_{0}^{1}}^{5}\right) .
\end{aligned}
$$

We know that

$$
w=\left(k+k_{1}\right) h+O\left(k^{2}+k_{1}^{2}\right)=\left(k+k_{1}\right) h+O\left(\left(k+k_{1}\right)^{2}\right) .
$$

Thus,

$$
\begin{aligned}
\bar{\Delta}= & \frac{1}{2} \bar{I}_{t\left(s_{0}+k\right)}^{\prime \prime}\left(u\left(s_{0}-k_{1}\right)\right) \cdot h \cdot h\left(k+k_{1}\right)^{2} \\
& +\frac{1}{2} \bar{I}_{t\left(s_{0}+k\right)}^{\prime \prime}\left(u\left(s_{0}-k_{1}\right)\right) \cdot h \cdot O\left(\left(k+k_{1}\right)^{2}\right)\left(k+k_{1}\right) \\
& +O\left(\left(k+k_{1}\right)^{4}\right)+\frac{1}{6} \bar{I}_{t\left(s_{0}+k\right)}^{(3)}\left(u\left(s_{0}-k_{1}\right)\right) \cdot h \cdot h \cdot h\left(k+k_{1}\right)^{3} \\
= & \frac{1}{2} \bar{I}_{t\left(s_{0}\right)}^{\prime \prime}\left(u\left(s_{0}\right)\right) \cdot h \cdot h\left(k+k_{1}\right)^{2}+\left.\frac{\dot{t}\left(s_{0}\right)}{2} k \frac{\partial}{\partial t} \bar{I}_{t}^{\prime \prime}\left(u\left(s_{0}\right)\right) \cdot h \cdot h\left(k+k_{1}\right)^{2}\right|_{t=t\left(s_{0}\right)} \\
& +O\left(\left(k+k_{1}\right)^{4}\right)+\frac{1}{2}\left(\bar{I}_{t\left(s_{0}+k\right)}^{\prime \prime}\left(u\left(s_{0}-k_{1}\right)\right)-\bar{I}_{t\left(s_{0}+k\right)}^{\prime \prime}\left(u\left(s_{0}\right)\right)\right) \cdot h \cdot h\left(k+k_{1}\right)^{2} \\
& +\frac{1}{6} \bar{I}_{t\left(s_{0}\right)}^{(3)}\left(u\left(s_{0}\right)\right) \cdot h \cdot h \cdot h\left(k+k_{1}\right)^{3}+O\left(\left(k+k_{1}\right)^{4}\right) \\
= & \frac{1}{2} \bar{I}_{t\left(s_{0}\right)}^{(3)}\left(u\left(s_{0}\right)\right) \cdot h \cdot h \cdot h\left(k+k_{1}\right)^{2} \cdot\left(-k_{1}\right) \\
& +\frac{1}{6} \bar{I}_{t\left(s_{0}\right)}^{(3)}\left(u\left(s_{0}\right)\right) \cdot h \cdot h \cdot h\left(k+k_{1}\right)^{3}+O\left(\left(k+k_{1}\right)^{4}\right) .
\end{aligned}
$$

On the other hand,

$$
t\left(s_{0}+k\right)=t\left(s_{0}\right)+\frac{1}{2} t^{\prime \prime}\left(s_{0}\right) k^{2}+O\left(k^{3}\right), \quad t\left(s_{0}-k_{1}\right)=t\left(s_{0}\right)+\frac{1}{2} t^{\prime \prime}\left(s_{0}\right) k_{1}^{2}+O\left(k_{1}^{3}\right),
$$


so that, since $t\left(s_{0}+k\right)=t\left(s_{0}-k_{1}\right)$,

$$
k=k_{1}(1+o(1)) .
$$

Thus

$$
\bar{\Delta}=-\frac{1}{12} \bar{I}_{t\left(s_{0}\right)}^{(3)}\left(u\left(s_{0}\right)\right) \cdot h \cdot h \cdot h\left(k+k_{1}\right)^{3}+O\left(\left(k+k_{1}\right)^{4}\right) .
$$

We set $t\left(s_{0}\right)=1$ so that

$$
\bar{\Delta}=\frac{1}{12} \int g^{\prime \prime}\left(u\left(s_{0}\right)\right) h^{3}\left(k+k_{1}\right)^{3}+O\left(\left(k+k_{1}\right)^{4}\right) .
$$

Differentiating $(*)$, we derive (at $\left.s_{0}\right)$

$$
\left\{\begin{array}{l}
-\Delta \dot{h}-g^{\prime}(u) \dot{h}=g^{\prime \prime}(u) h^{2}, \\
\dot{h}+\left.r(\sigma) \ddot{t}\left(s_{0}\right) \frac{\partial u_{t}}{\partial r}(\sigma, \operatorname{tr}(\sigma))\right|_{\partial \Omega_{t\left(s_{0}\right)}}=0 .
\end{array}\right.
$$

Thus,

$$
\begin{aligned}
\int g^{\prime \prime}(u) h^{3} & =\int_{\Omega_{t\left(s_{0}\right)}}\left(-\Delta \dot{h}-g^{\prime}(u) \dot{h}\right) h=\int \nabla \dot{h} \nabla h-\int g^{\prime}(u) h \dot{h} \\
& =\int_{\partial \Omega_{t\left(s_{0}\right)}} \dot{h} \frac{\partial h}{\partial v}-\int_{\Omega}\left(\Delta h+g^{\prime}(u) h\right) \dot{h}=\int_{\partial \Omega_{t\left(s_{0}\right)}} \dot{h} \frac{\partial h}{\partial v} \\
& =-\ddot{t}\left(s_{0}\right) \int_{\partial \Omega_{t\left(s_{0}\right)}} \frac{\partial u_{t}}{\partial r} \frac{\partial h}{\partial v} r(\sigma) d \sigma_{t}=-\ddot{t}\left(s_{0}\right) \int_{\partial \Omega_{t\left(s_{0}\right)}} \frac{\partial u_{t\left(s_{0}\right)}}{\partial v} \frac{\partial h}{\partial v} x \cdot v d \sigma_{t} .
\end{aligned}
$$

On the other hand, at every $t$,

$$
\int_{\partial \Omega_{t\left(s_{0}\right)}}\left|\frac{\partial u_{t}}{\partial v}\right|^{2} x \cdot v d \sigma_{t}=c_{n}\left(\int_{\Omega_{t\left(s_{0}\right)}} g(u) u-\frac{n-2}{2 n} G(u)\right) .
$$

Differentiating and applying at $s=s_{0}$, we find $\left(\dot{t}\left(s_{0}\right)=0\right)$

$$
2 \int_{\partial \Omega_{t\left(s_{0}\right)}} \frac{\partial h}{\partial v} \frac{\partial u_{t}}{\partial v} x \cdot v d \sigma_{t}=c_{n} \int_{\Omega_{t}}\left(\frac{n+2}{2 n} g(u) h+g^{\prime}(u) u h\right)=\bar{c}_{n} \int_{\Omega_{t}\left(s_{0}\right)} g(u) h .
$$

Thus, at $s_{0}$,

$$
\int g^{\prime \prime}(u) h^{3}=-\frac{\bar{c}_{n}}{2} \ddot{t}\left(s_{0}\right) \int_{\Omega_{t\left(s_{0}\right)}} g(u) h .
$$

We see that the sign of $\int g^{\prime \prime}(u) h^{3}$ depends on $\ddot{t}\left(s_{0}\right)$ and on $\int_{\Omega_{t\left(s_{0}\right)}} g(u) h$. Thus, the change of the Morse index at the crossing of $t\left(s_{0}\right)$ depends on the convexity of $t(s)$ and on the sign of $\int_{\Omega_{t^{\left(s_{0}\right)}}} g(u) h$. This is directly related to the rotation of $\dot{t}(s)+i \int_{\Omega_{t(s)}} g(u) h$, which in turn relates directly to $\dot{I}+i \dot{\int \bar{G}}$, hence to $\overline{\left.\int \dot{\nabla} u\right|^{2}}+i \dot{\int \bar{G}}$. Theorem 1 follows. 


\section{References}

[1] Harrabi, A., Rebhi, S., Selmi, A.: Solutions of superlinear elliptic equations and their Morse indices, I, II. Duke Math. J. 94, 141-157, 159-179 (1998) Zbl 0952.35042 MR 1635912

[2] Yang, X. F.: Nodal sets and Morse indices of solutions of superlinear elliptic PDEs. J. Funct. Anal. 160, 223-253 (1990) Zbl 0919.35049 MR 1658692 